\title{
DOMINGO DE SOTO E LA FONDAZIONE DELLA SCUOLA DI SALAMANCA
}

Merio Scattola*

RESUMO - Na história do pensamento político e do direito, podemos considerar ou seus conteúdos ideológicos ou o modo de comunicá-los. Se reconstruirmos a história da Segunda Escolástica no segundo sentido, podemos avaliar a contribuição de Domingo de Soto, que organizou todo o tema em um sistema claro e apresentou com seu tratado Sobre a justiça e o direito uma forma literária de debate bem-sucedida. Nesse sentido, Soto merece ser considerado um fundador da Segunda Escolástica junto com Francisco de Vitoria. Relevante é o esquema que ele adota na distribuição topológica, como a aproximação por ele realizada entre as noções de direito e justiça mediante a doutrina da liberdade e domínio, que figurou como um verdadeiro ponto de partida para a teoria do direito, especialmente para uma teoria do direito natural.

PALAVRAS-CHAVE - História do pensamento político. Filosofia do direito. Teoria da justiça. Direito natural. Segunda Escolástica. Escola de Salamanca. Teoria da propriedade.
ABSTRACT - In the history of political and legal thought we can consider either the ideological contents or the way of communicating them. If we reconstruct the history of Late Scholasticism in this second sense, we can appraise the contribution of Domingo de Soto, who organized the entire scholastic matter in a clear system and introduced with his treatise On justice and law a successful literary form of discussion. In this sense, Soto deserves to be considered a founder of Late Scholasticism together with Francisco de Vitoria. Relevant is the scheme he adopted in the topological distribution, as he joined the notions of law and of justice through a doctrine of freedom and dominion, which figured as a true starting point for a theory of law, especially for a theory of natural law.

KEY WORDS - History of political thought. Philosophy of law. Theory of justice. Natural law. Late Scholasticism. School of Salamanca. Theory of property.

\footnotetext{
* Università di Padova.
}

\begin{tabular}{|l|l|l|l|l|l|}
\hline VERITAS & Porto Alegre & v. 54 & n. 3 & set./dez. 2009 & p. 52-70 \\
\hline
\end{tabular}




\section{Storia delle idee politiche e storia dei generi letterari}

Fin dalla sua nascita la storia delle idee politiche e giuridiche è stata concepita come una storia di "contenuti", cioè di concezioni, filosofie o ideologie, che nel corso dei secoli sono state utilizzate per sostenere determinate interpretazioni della vita sociale e per combattere altre visioni rivali. Tutte le scuole, la Ideengeschichte di Friedrich Meinecke o la History of ideas di Arthur Lovejoy, la storia del linguaggio politico di Quentin Skinner o la Begriffsgeschichte di Reinhart Koselleck, convengono sulle caratteristiche generali di questo processo e divergono soprattutto quando stabiliscono quale sia la natura propria di queste idee: se esse corrispondano a un principio fondamentale della vita statuale ${ }^{1}$ oppure a una pluralità di elementi irriducibili e combinati in modi infiniti, ${ }^{2}$ se esse siano strutture costitutive delle epoche storiche, ${ }^{3}$ o se debbano andare interpretate nel rapporto tra grammatica e realizzazione, nello stesso modo in cui la linguistica concepisce il nesso tra langue e parole. ${ }^{4} \mathrm{Nulla}$ togliendo ai risultati che si possono raggiungere con le metodologie tradizionali basate sui contenuti, si può tuttavia tentare anche l'altra strada, quella che guarda alle forme del discorso politico e giuridico.

Guardando soprattutto ai contenuti, definiamo "Seconda Scolastica" o "Tarda Scolastica" quel gruppo di teologi, soprattutto domenicani e gesuiti, che, seguendo l'esempio di Francisco de Vitoria (ca.14921546), ripresero l'opera di Tommaso d'Aquino per riformulare la teologia cattolica nell'epoca della Riforma. ${ }^{5}$ Ugualmente si parla di una Scuola di Salamanca, intendendo così quei teologi spagnoli che utilizzarono i materiali della tradizione tomista per comprendere i problemi sollevati dalla scoperta dell'America. ${ }^{6}$ In questi casi possiamo tuttavia chiederci se quei gruppi fossero uniti solo dall'interesse per certi principi, o se essi invece utilizzassero anche una particolare forma della conoscenza scientifica, privilegiando certi generi letterari anzi che altri e sfruttando particolari strategie di comunicazione.

Considerato da questo punto di vista, il pensiero politico europeo del Cinquecento e del Seicento ci offre alcune sorprese. Esso è chiaramente diviso in aree confessionali e vagamente nazionali, le quali tuttavia non sembrano essere caratterizzate dalla presenza di dottrine specifiche, anzi i contenuti sembrano muoversi senza ostacoli sui territori europei

\footnotetext{
MEINECKE. 1977, p. 1-22. Cfr. SCATTOLA. 2006a, p. 167-206.

LOVEJOY. 1966, p. 11-29; ID. 1982, p. 29-41. Cfr. ROSSI. 1982, p. 7-17.

KOSELLECK. 1972, p. XIII-XXVII. Cfr. SCATTOLA. 2006b, p. 95-124.

SKINNER. 1969, p. 3-53; ID. 2002, p. 57-89.

GIACON. 1950.

PEREÑA VICENTE. 1954; ID. 1986; ID. 1981, p. 27-94.
} 
e formare una sorta di patrimonio comune condiviso liberamente. Per esempio, non è possibile attribuire dottrine così caratteristiche come quelle della tirannide e del diritto di resistenza, a questa o a quella confessione, perché esse furono utilizzate indifferentemente da cattolici, luterani e calvinisti. ${ }^{7}$ Le comunità del discorso politico sembrano semmai organizzarsi e distinguersi l'una dall'altra più rispetto ai loro stili di comunicazione che alle dottrine difese. ${ }^{8}$ Se per esempio consideriamo l'Italia, troviamo prevalentemente scrittori attivi nelle corti e nelle cancellerie come segretari, consiglieri, funzionari, agenti, talvolta spie, ${ }^{9}$ i quali praticavano il genere letterario dei discursus, un plurale con il quale si indicava una trattazione abbastanza libera, non vincolata agli schemi accademici e dedicata a temi spesso scabrosi, come potevano essere la ragion di stato, gli arcana imperii e in generale tutte le dottrine della deroga. ${ }^{10}$

Il discorso politico durante il regno di Elisabetta I d'Inghilterra era invece caratterizzato da un'attenzione quasi esclusiva sulle transazioni simboliche e dalla marginalizzazione del discorso dottrinale, ${ }^{11}$ nelle forme della filosofia e della giurisprudenza, che conquistò invece centralità con Giacomo I e con le polemiche sul giuramento di fedeltà alla corona e sul primato del re. ${ }^{12}$

Anche per il pensiero politico e giuridico spagnolo, per la Seconda Scolastica del Cinquecento, una lettura in questa prospettiva formale può produrre rilevanti sorprese. La prima sorpresa è che, se guardiamo più alla forma che ai contenuti, il vero fondatore della scuola di Salamanca non è Francisco de Vitoria, ma Domingo de Soto (1494-1560).

\section{Lo stile di Salamanca}

Jaime Brufau Pratt ha mostrato come la tradizionale visione, secondo cui al maestro Vitoria succederebbe l'allievo Soto, può essere capovolta o, almeno, attenuata, se si mettono a confronto le lezioni di Vitoria sulla Secunda secundae del 1535 e la Relectio de dominio di Soto, che fu

7 SCATTOLA. 1996, p. 391-420.

8 SCATTOLA. 2002-2003, p. 5-8; ID. 2006c, p. 22-55.

9 COMPARATO. 1999, p. 133-142; BALDINI. 1995, p. 465-482.

10 COLER, CH. "De studio politico ordinando epistola". 1602, p. 117; BOSE, J. A. De comparanda prudentia iuxta et eloquentia civili deque libris et scriptoribus ad eam rem maxime aptis dissertationes isagogicae (1677). Ienae, Bielkius, 1678, p. 38-39. Per una definizione del genere dei discursus cfr. BORNITZ, J. Discursus politicus de prudentia politica comparanda, Erphordiae, Birnstilius, 1602, f. A4r. Cfr. ZWIERLEIN. 2006.

11 YATES. 1975; GUY. 1995; MEARS. 2005.

12 GREENLEAF. 1957; CAVARERO. 1980; BINGHAM. 1981. 
pronunciata qualche mese prima, nello stesso anno $1535 .{ }^{13}$ Anche senza ricorrere a quest'argomentazione, l'importanza di Soto rimane indubbia se si considera quanto egli abbia contribuito a costruire l'identità della Seconda Scolastica.

Se vogliamo descrivere il pensiero politico spagnolo del quindicesimo secolo, possiamo dire che si trattava di una dottrina e di una prassi politica elaborate in primo luogo da e per teologi e canonisti, la quale può bene essere simboleggiata con l'unione di cattedra, pulpito e confessionale. L'università di Salamanca era infatti il luogo di formazione dei funzionari di rango superiore, che sostenevano la monarchia nel governo dei regni iberici; ${ }^{14}$ essa concepiva perciò il politico fondamentalmente ed esclusivamente come il consulente, il consigliere di corte o il ministro e sviluppò una forma di pensiero politico che coincideva con questa figura e con la sua formazione. Aprendo le sue Decisiones de iustitia et iure Domingo Báñez (1528-1604) nota che l'interesse per le materie della giustizia è comune a filosofi, giuristi e teologi e, chiedendosi a quale facoltà spetti trattare questi argomenti, osserva che essi toccano alla filosofia, nella misura in cui questa considera il fine naturale dell'uomo, ma pertengono alla giurisprudenza se si considerano le leggi umane, e infine sono da rivendicarsi propriamente alla teologia perché riguardano il fine soprannaturale e la salvezza eterna dell'anima. ${ }^{15}$

Il politico era dunque teologo, giurista e confessore e agiva prevalentemente con gli strumenti della teologia morale, che erano "scolastici" nei due sensi del termine. In un primo senso, tutti i generi letterari sviluppati da questa tradizione erano infatti mezzi per propagare il sapere all'interno della scuola. L'unità fondamentale era la quaestio, che poteva avere due sviluppi: uno quantitativo e uno qualitativo. Le questioni potevano infatti essere moltiplicate e riunite in fasci, e allora davano origine al tractatus, alla summa o ai loci communes, come nel caso di Melchor Cano (1509-1560). ${ }^{16}$ La questione poteva tuttavia anche essere mantenuta isolata, intensificando la sua qualità, stilistica o argomentativa, e in tal caso la lectio si trasformava nella relectio, la lezione ufficiale e cerimoniale, tenuta in particolari occasioni di fronte all'intero corpo accademico. Ma tutti questi generi erano "scolastici"

13 DOMINGO de Soto. Relección De dominio, par. 36, p. 162-164; FRANCISCO de Vitória. De iustitia. Tomo primero, IIa IIae, quaest. 62, art. 1, p. 81-82. Cfr. BRUFAU PRATS. 1964a, p. XXVII.

14 REIBSTEIN. 1955; PEREÑA VICENTE. 1954, p. 72-92; ID. 1986, p. 55-60.

15 DOMINGO Báñez. Decisiones de iure et iustitia, in IIa IIae, quaest. 57, Prologus auctoris, p. 1 ${ }^{\text {b }}$. Cfr. CRUZ CRUZ. [2008], p. 29-30.

16 CANO, M. De locis theologicis libri duodecim, Salmanticae, Gastius, 1563. 
anche in un secondo senso perché si riferivamo alla Schola per eccellenza, all'insegnamento di Tommaso d'Aquino.

In questo schema Vitoria praticò il commento alla Summa theologiae nelle sue lezioni, ma affidò la sua fama alle relectiones, concepite per un pubblico esterno, ma dedicate ad argomenti particolari e perciò occasionali. L'elaborazione sistematica della materia scolastica in un unico corpo disciplinare, l'elaborazione di un "sistema" scolastico, spettò invece a Domingo de Soto, che propose il primo trattato De iustitia et iure e inventò il genere letterario con il quale si identificò la discussione della Seconda Scolastica. In tal senso l'impatto del suo trattato fu uguale o forse maggiore delle stesse Relectiones di Vitoria. Tra le opere che trovarono maggiore eco nei dibattiti europei di fine Cinquecento, più che le Relectiones di Vitoria, troviamo infatti le Controversiae illustres di Fernando Vázquez Menchaca, le Variae resolutiones di Diego de Covarrubias y Leyva e i De iustitia et iure libri decem di Domingo de Soto, ${ }^{17}$ tre casi che introdussero nuovi generi letterari o che portarono forme già esistenti al massimo livello di perfezione formale. ${ }^{18}$

\section{II trattato teologico-giuridico}

Nella storia delle ricezione europea, se si eccettuano le trattazioni speciali del diritto di guerra, come i trattati di Hugo Grotius (1583-1645), ${ }^{19}$ il primo grande esponente della scuola di Salamanca, più che Vitoria, sembra essere Soto, che diede alla scuola di Salamanca il suo strumento di indagine caratteristico e il suo "sistema".

Anche Francisco de Vitoria aveva commentato la dottrina di Tommaso d'Aquino nel suo secondo corso sulla Secunda secundae degli anni 1534-1536, ma aveva seguito fedelmente lo schema tomista trattando la

17 Cfr. ARNISAEUS, H. De iure maiestatis libri tres, I, 2, p. 25 (Soto), 29-30, 33 (Covarrubias, Vázquez); I, 3, p. 43, 50 (Covarrubias, Vázquez); ALTHUSIUS, I. Politica methodice digesta atque exemplis sacris et profanis illustrata, p. 38, 37, 897 (Soto), 78-129, p. 917-936 (Vázquez, Covarrubias); ARNISAEUS, H., Index autorum, f. b4v (Soto); MEISNER, B. Dissertatio de legibus, in quatuor libellos distributa, quorum primus agit de lege in genere, secundus de lege aeterna, tertius de lege naturae, quartus de legibus humanis, tum politicis tum ecclesiasticis, III, 2, p. 214-7 (Soto); CONTZEN, A. Politicorum libri decem, Catalogus auctorum, f. ** 5r, V, 1, p. 302 (Soto); UNGEPAUR, E. Exercitia Iustinianea, sive disputationes ad IV. libros Institutionum imperatoris Iustiniani, 2,1, p. 45-49 (Soto).

18 COVARRUBIAS Y LEYVA, D. DE. Regulae. Peccatum. De regulis iuris, lib. 6. Relectio (1554); ID. Variarum ex iure pontificio, regio et Caesareo resolutionum libri III (1552).; VÁZQUEZ MENCHACA, F. Controversiarum illustrium aliarumque usu frequentium libri tres (1564).

19 GROTIUS, H. De iure praedae commentarius (1604-1605), p. 84 (Soto), 25, 29, 48, 57, 62, 67 (Vitoria), 57 (Covarrubias); FEENSTRA; PERSENAIRE. 1993, p. 1064 (Soto), 1069-1069 (Vitoria); THIEME. 1986, Bd. 2, p. 910-911. 
sequenza delle virtù umane senza riferimento alle materie della Prima secundae. ${ }^{20}$ Con Domingo de Soto iniziò invece una revisione della topica tomista che nella seconda metà del secolo andò progressivamente accelerando nelle opere di Gabriel Vázquez, Luis de Molina e Francisco Suárez, che effettivamente abbandonarono la forma canonica del commento.

Nei suoi De iustitia et iure libri decem Domingo de Soto perseguì esplicitamente l'obiettivo di dare sistemazione al sapere scolastico, soprattutto alla luce dei nuovi istituti introdotti nel campo della giurisprudenza, come cambiali, girate, assegni e ordini di pagamento, che, a suo dire, avevano stravolto le relazioni morali tra gli uomini. ${ }^{21} \mathrm{La}$ conservazione dei buoni rapporti entro la società umana richiedeva in primo luogo la riforma del sapere giuridico e politico per determinare i confini del lecito e dell'illecito, alla luce delle conoscenze raccolte dalla teologia, dalla giurisprudenza e dalla filosofia. Non si trattava perciò di scoprire e introdurre una nuova forma di sapere, ma di riorganizzare nel modo corretto le conoscenze messe a disposizione dalla tradizione scolastica, la res scholastica. ${ }^{22}$

Per la sua sistemazione Soto scelse tutte le parti della Summa theologiae relative al diritto e unì così il trattato De legibus, comprendente le questioni 90-109 della Prima secundae, con il trattato De iustitia, che include le questioni 57-122 della Secunda secundae. Non si trattò tuttavia di una semplice giustapposizione di commenti, ottenuta, per così dire, sottraendo i materiali intermedi che separano questi due grandi blocchi argomentativi. La sequenza dello schema tomista viene infatti in più punti perturbata per ottenere una maggiore coerenza nella successione degli argomenti, nuovi complessi tematici sono inseriti in punti nodali della trattazione e interi comparti, assenti nel testo commentato, completano il trattato come un grande aggiornamento in appendice.

Lo schema utilizzato da Soto distingue innanzi tutto il diritto in senso proprio dal diritto in senso traslato. In senso proprio il diritto può essere inteso come norma che regola il comportamento umano oppure come concreto rapporto tra persone. Nel primo caso si ha la legge, che si articola in quattro differenti specie (eterna, naturale, umana e divina), nel secondo caso la giustizia. Quest'ultima può essere distributiva o commutativa, a

20 BELTRÁN DE HEREDIA. 1934, p. IX-XII.

${ }^{21}$ DOMINGO de Soto. De iustitia et iure libri decem, lib. VI, p. 505a-610 b. Cfr. BARRIENTOS GARCIA. 1985, p. 139-278.

22 DOMINGO de Soto. De iustitia et iure libri decem, lib. I, Prooemium, p. 5 $5^{\mathrm{a} b}$. Soto propose un'identica riorganizzazione del sapere scolastico anche nel campo della logica. Cfr. DOMINGO de Soto. Summulae; RISSE. 1980; MUÑOZ DELGADO. 1964, p. 27-59. 
seconda che essa regoli i rapporti della comunità con le sue parti ovvero delle parti tra di loro. ${ }^{23} \mathrm{~A}$ sua volta la giustizia commutativa può essere involontaria, quando interviene per riparare una violazione, o volontaria, quando gli uomini stringono rapporti di scambio contrattuale. In senso lato, il diritto coincide con le virtù accessorie alla giustizia, classificate in vario modo dall'antichità, ${ }^{24}$ e da Soto ridotte alla triade di religione, generosità e misericordia.

Alla discussione delle leggi Soto dedica i primi due libri del suo trattato, laddove il primo tratta i primi tre tipi di legge: eterna, naturale e umana (Ia IIae, quaest. 90-97), mentre l'intero secondo libro è dedicato alla sola legge divina e commenta le questioni 98-109. Con il terzo libro inizia la discussione del trattato tomista De iustitia, che nelle questioni 57-63 chiarisce le questioni generali del diritto. Soto utilizza la tradizionale distinzione aristotelica tra giustizia distributiva e giustizia commutativa, ${ }^{25}$ discussa da Tommaso d'Aquino nella questione 61 della Secunda secundae, presentando brevemente la prima delle due forme.

Con il quarto libro inizia la trattazione della giustizia commutativa, che si estende per tre libri e che culmina nella discussione dei generi moderni di transazione economica. Questa parte costituisce un blocco argomentativo molto compatto, reso ancora più omogeneo dall'intervento di Soto che riorganizza a fondo la distribuzione tomista. Tommaso d'Aquino aveva introdotto la giustizia commutativa discutendo della riparazione, in modo da considerare l'intera giustizia penale come un processo di commisurazione tra azioni, ${ }^{26}$ e aveva quindi applicato la divisione aristotelica tra rapporti di scambio non volontari e volontari, ${ }^{27}$ ripercorrendo vagamente l'elenco dei delitti indicato da Aristotele. Soto riconosce qui la necessità di rendere ancora più evidenti i presupposti della giustizia commutativa, rimasti inespressi nella trattazione tomista. Se questo genere di giustizia si basa sullo scambio di beni o di azioni secondo un rapporto di equivalenza, affinché la cessione abbia luogo, è necessario in primo luogo che i soggetti coinvolti detengano il possesso delle azioni o dei beni che trasferiscono. L'esposizione della giustizia commutativa deve perciò cominciare dalla dottrina del dominio, con la quale si dimostra che un essere umano può scambiare una cosa o un'azione perché è libero dal dominio altrui. "Il dominio sulle cose e la loro divisione è quindi la base e il fondamento di tutti i contratti, gli accordi e i patti che vengono stipulati attraverso la giustizia

23 TOMÁS de Aquino. Summa theologiae, IIa IIae, quaest. 61, art. 1, p. $1348^{\mathrm{b}}$.

24 TOMÁS de Aquino. Summa theologiae, IIa IIae, quaest. 80, art. 1, p. $1415^{\mathrm{a}-\mathrm{b}}$.

25 ARISTOTELES. Ethica Nicomachea, lib. V, cap. 2, 1130 $30-34$.

26 BETTETINI. 1994.

27 ARISTOTELES. Ethica Nicomachea, lib. V, cap. 2, 1131ª 1-9. 
commutativa". ${ }^{28}$ Soto perciò inserisce all'inizio del quarto libro un lungo trattato in cinque parti sul dominio, che recupera molti temi affrontati nell'omonima relectio del $1535,{ }^{29}$ e riprende poi lo schema tomista discutendo nella seconda parte del libro il tema della restituzione. ${ }^{30} \mathrm{Il}$ quinto libro analizza quindi le specie involontarie di giustizia commutativa, che comprendono la violenza verso le persone (omicidio e ferimento), verso i beni materiali (furto), i comportamenti iniqui delle parti coinvolte in un processo (giudice, accusatore, reo, testimoni e avvocati) e le offese della buona fama (insolenza, insulto, derisione, maledizione).

Il sesto libro espone le materie della giustizia commutativa nei rapporti spontanei, quali sono tutte le forme di contratto esplicito, e toccando così gli argomenti della finanza già ricordati nell'introduzione realizza il vero fine del trattato. ${ }^{31}$ Alla discussione di questi problemi Tommaso d'Aquino aveva riservato due sole questioni, la settantasettesima e settantottesima, dedicate alla frode e all'usura, una descrizione evidentemente troppo limitata e per di più incongruamente disposta per due temi tanto centrali nella vita morale e politica del secolo XVI. Soto inverte perciò l'ordine di presentazione e tratta prima l'usura, con cui si vende ciò che non ha prezzo, e poi la frode, con la quale si scambia un bene per un prezzo ingiusto, e integra la discussione con gli istituti non conosciuti o non menzionati da Tommaso d'Aquino: la vendita a credito, la compravendita del censo, il contratto di società, il contratto di assicurazione, il cambio e la compravendita di moneta.

Con il settimo libro inizia l'esposizione delle "parti potenziali della giustizia", con cui si intendono tutte quelle virtù che non fanno parte della giustizia secondo la relazione che unisce specie a generi, ma che tuttavia hanno con essa un rapporto di tipo topologico, così che possono essere ricondotte a essa come al loro punto di riferimento unificatore. Soto semplifica le classificazioni antiche delle virtù accessorie, ${ }^{32}$ che anche da Tommaso d'Aquino erano state fissate nel numero di nove, ${ }^{33}$ e riduce le parti potenziali a soli tre abiti: la religione, la generosità e la

28 DOMINGO de Soto. De iustitia et iure libri decem, lib. V, Prooemium, p. 278a.

29 DOMINGO de Soto. Relección De dominio; BRUFAU PRATS. 1989a, p. 11-47; ID. 1964b, p. 1-62; ID. 1989b, p. 79-101.

30 DOMINGO de Soto. De iustitia et iure libri decem, lib. V, Prooemium, p. 278a.

31 DOMINGO de Soto. De iustitia et iure libri decem, lib. VI, Prooemium, p. 505a: "Eo denique destinati operis perventum nobis est, cuius praecipue gratia de illo coepimus cogitare. Haec inquam usurarum contractuum cambiorumque ac simoniarum sylva in animum potissime nos induxit, ut tantam operis molem aggrederemur. Videmus enim instantia tempora huiusmodi iniustitiae avaritiaeque portentis usque adeo scaturire, ut vix reliqua nobis fiat spes veritatem persuadendi".

32 TOMÁS de Aquino. Summa theologiae, IIa IIae, quaest. 80, art. 1, p. $1415^{\mathrm{a}-\mathrm{b}}$.

33 TOMÁS de Aquino. Summa theologiae, IIa IIae, quaest. 81, art. 1, p. $1417^{\mathrm{a}-\mathrm{b}}$. 
misericordia ${ }^{34}$ Rinuncia tuttavia a una discussione esauriente e dedica gli ultimi quattro libri del suo trattato alla sola religione, presentando alcuni dei temi all'ordine del giorno nelle discussioni del Concilio di Trento, cui egli partecipò direttamente dal 1545 al 1547. ${ }^{35}$ Il settimo libro tratta così della promessa in generale, della dispensa e in particolare dei voti sacerdotali, riprendendo le materie discusse da Tommaso nella ottantottesima questione. L'ottavo libro è interamente occupato da un libellus de iureiurando, ${ }^{36}$ che espone le materie del giuramento e dello spergiuro, corrispondenti alle questioni 89 e 98 della Secunda secundae, mentre il nono libro mostra quali beni siano dovuti a Dio, come l'ostia, le primizie e le decime, e si sofferma in modo particolare sul peccato di simonia (questioni 85, 86 e 100). ${ }^{37} \mathrm{Il}$ decimo e ultimo libro affronta infine il problema della residenza dei vescovi nelle diocesi, un tema propriamente tridentino ed estraneo alla Summa theologiae di Tommaso d'Aquino.

Considerato da una certa distanza, il trattato di Soto si divide in due grandi parti, la prima delle quali comprende nei primi sei libri, quello che potremmo chiamare il "complesso filosofico-giuridico", mentre gli ultimi quattro libri trattano materie canonistiche secondo un ordine di tipo topologico. In entrambi i casi Soto si richiama allo schema scolastico di Tommaso d'Aquino per migliorarne l'evidenza e l'efficacia argomentativa, cioè per dimostrare che esso può organizzare il più alto numero di dottrine anche quando queste sembrano contraddirsi.

Allo stesso progetto di organizzare con un piano topologico coerente le materie giuridiche della tradizione scolastica, parteciparono anche tutti gli altri esponenti della tradizione scolastica spagnola, che proseguirono il lavoro avviato da Soto e arricchirono lo schema tomista, mentre allo steso tempo si allontanavano sempre più da esso. Così Gabriel Vázquez (1549-1604) concepì il suo commentario come una serie di disputazioni autonome, che seguivano la medesima sequenza impressa da Tommaso d'Aquino alle questioni della sua Summa theologiae, ma selezionavano drasticamente i temi trattati concentrandosi prevalentemente sui problemi della teologia morale. ${ }^{38}$ Negli stessi anni Luis de Molina (1535-1600) si scusò espressamente nel proemio al suo trattato De iustitia et iure di avere utilizzato una disposizione personale che di fatto trasformava il commento alla Summa theologiae in una successione di trattati

34 DOMINGO de Soto. De iustitia et iure libri decem, lib. VII, quaest. 1, art. 1, p. 611 a-b.

35 BELTRÁN DE HEREDIA. 1960, p. 117-173.

36 DOMINGO de Soto. De cavendo iuramentorum abusu, ad laudem divini nominis institutio. Cfr. DOMINGO de Soto. El abuso de los juramentos. La ocultación y revelación de secretos.

37 DOMINGO de Soto. De iustitia et iure libri decem, lib. VIII, Prooemium, p. 709a.

38 SCATTOLA. 2003, p. 11920; FELLERMEIER. 1940. 
autonomi. ${ }^{39}$ Infine Francisco Suárez (1548-1617) dissolse completamente l'ordinamento originario nella sua opera sulle leggi, adottando anche per le singole parti una nuova procedura in tre fasi che raccoglieva per ogni discussione prima le autorità scritturali, quindi le prove storiche e infine le argomentazioni razionali. ${ }^{40}$

\section{La dottrina del dominio}

In ciascuno dei punti cruciali del suo trattato, dove era necessario rendere evidente il nesso tra gli argomenti, Soto riorganizzò la materia scolastica ed elaborò schemi di interpretazione che confluirono nella tradizione successiva. Esempi rilevanti di quest'opera di sistemazione sono la dottrina della legge $\mathrm{e}^{41}$ oppure la discussione circa l'ammissibilità della dispensa per i comandi del diritto naturale. ${ }^{42} \mathrm{Un}$ altro punto nel quale è molto evidente la riorganizzazione operata da Domingo de Soto è la dottrina del dominio, che è particolarmente rilevante sia perché fu successivamente ripresa dagli altri autori della Seconda Scolastica ${ }^{43}$ sia perché la sua formulazione è avvolta da un alone di mistero. Su questo tema Soto pronunciò infatti una Relectio de dominio verosimilmente tra il 28 febbraio e il 24 giugno 1535 a commento del quarto libro delle Sentenze di Pietro Lombardo, ${ }^{44}$ ma anche Francisco de Vitoria, esplicando tra novembre e dicembre 1535 la questione 62 della Secunda secundae, trattò a fondo questi argomenti e in termini sostanzialmente simili. Soto infine incorporò parti cospicue della sua relectio del 1535 nel suo trattato De iustitia et iure del 1553-1554, conservandole sostanzialmente intatte. ${ }^{45}$

Apprestandosi a discutere della restituzione, la satisfactio o restitutio, Soto rileva che la questione non è risolvibile se non si chiarisce prima

39 LUÍS de Molina. De iustitia et iure opera omnia, tract. 1, Auctoris consilium, col. 1: "Si eam theologicae partem, quae de moribus disserit, copiose et pro dignitate et amplitudine obiecti et facultatis theologiae tradiderimus, ea, quae virum theologum ex iis, quae iuris periti tractant, scire decet, nec sine methodo et arte theologiae inseruerimus, nihil viro theologo deerit, quod ad ecclesiae gubernationem et reipublicae Christianae utilitatem necessarium fuerit iudicatum. Hoc consilio ductus multisque aliis gravissimis de causis permotus, ordinem divi Thomae in his 23 quaestionibus praeter nostrum morem relinquere opusque hoc de iustitia in varios tomos distributum earum loco inserere statui".

40 FRANCISCO Suárez. De legibus (II 1-12). De lege naturali.

41 SCATTOLA. 2001, p. 21-47.

42 DOMINGO de Soto. De iustitia et iure libri decem, lib. II, quaest. 3, art. 8, p. $114^{\text {b }}$ 120 ${ }^{\mathrm{b}}$, soprattutto 115a-118 ${ }^{\mathrm{a}}$. Cfr. TODESCAN. 1973; BERTELLONI. 2007.

43 Cfr. per esempio VÁZQUEZ MENCHACA. Controversiarum illustrium aliarumque usu frequentium libri tres, vol. 1, lib. I, cap. 17, par. 8, p. 324.

44 BRUFAU PRATS. 1964b, p. 57; PETRUS LOMBARDUS. Sententiarum libri quatuor. lib. IV, dist. 16: De tribus quae in poenitentia consideranda sunt, col. 877-879.

45 BELTRÁN DE HEREDIA. 1934, p. X-XI. 
che cosa sia il dominio, perché possiamo comunicare beni e azioni solo se essi sono a nostra disposizione. ${ }^{46}$ Egli distingue in primo luogo due accezioni del termine "dominio", che può essere inteso come dominatio aut dominatus, cioè come comando politico connesso a una qualche forma di imperium, oppure come potestas seu ius che gli uomini hanno sulle cose. ${ }^{47}$ Quest'ultimo è anche il senso nel quale la nozione viene utilizzato sia da teologi e giuristi sia dallo stesso Soto, e che fondamentalmente indica il diritto di proprietà. Per rendere ragione della sua ampia casistica, il dominio deve esser definito come potestà o facoltà di assumere qualcosa per la propria utilità, con cui si comprendono sia la proprietà piena sia la nuda proprietà sia l'usufrutto sia l'uso. ${ }^{48}$ In sostanza il dominio è la possibilità di riferire alcune cose ed, eventualmente, anche alcune persone al proprio vantaggio, subordinandole alla propria volontà ed estendendo in tal modo lo spazio di azione del proprio corpo o costituendo un settore nell'universo dipendente dal proprio arbitrio.

Nella dottrina del diritto la nozione di dominio è rilevante per due aspetti. In primo luogo essa è il complementare della nozione di libertà, giacché solamente chi è libero può esercitare il dominio. "Sed homo per libertatem est dominus". ${ }^{49}$ D'altra parte vale anche la conclusione inversa, perché veramente libero è soltanto chi può esercitare il dominio su se stesso. Per conservare la vita, lo schiavo ha ceduto la sua libertà, e con ciò ha accettato di diventare uno strumento a servizio del suo padrone..$^{50}$ $\mathrm{Ma}$ in tal modo egli ha perso la capacità di determinare il proprio bene, anzi ha perso del tutto il proprio bene, eccettuando solo la conservazione della vita; egli non ha più dominio su di sé, ma ha ceduto tale facoltà, che gli spettava naturalmente in quanto nato libero, al suo padrone. Di conseguenza non può entrare in nessun altro rapporto che implichi la disposizione su di sé ed è perciò escluso sia dalle relazioni giuridiche sia dalla relazione politica.

Una dottrina delle relazioni politiche e, latamente, giuridiche deve perciò sempre cominciare stabilendo la condizione umana della libertà, che a sua volta è definibile solo attraverso una dottrina del dominio, come effettivamente è il caso sia della Politica di Aristotele sia delle Istituzioni

46 DOMINGO de Soto. Relección De dominio, Prooemium, p. 70.

47 DOMINGO de Soto. Relección De dominio, Accurata dominii notio declaratur, par. 41 p. 74.

48 DOMINGO de Soto. Relección De dominio, Accurata dominii notio declaratur, par. 2, p. 78.

49 DOMINGO de Soto. Relección De dominio, An iuste homo hominis dominus esse possit, par. 25, p. 128.

50 DOMINGO de Soto. Relección De dominio, An iuste homo hominis dominus esse possit, par. 25, p. 132. Cfr. TOMÁS de Aquino. In primum[-quartum] Sententiarum, to. 1, lib. IV, dist. 36, quaest. 1, art. 4, co. Cfr. SCATTOLA. 2007. 
di Giustiniano. In quest'ultimo caso la libertà o il dominio di sé sono infatti definiti negativamente, tanto che il libero è presentato come il non servo. ${ }^{51}$ "Infatti, alcune persone sono giuridicamente autonome e alcune sono soggette a potere altrui; ulteriormente, di quelle soggette a potere altrui, alcune sono in potestà di ascendenti, altre in potestà di padroni. Vediamo dunque quelle che sono soggette a potere altrui: perché una volta appreso quali siano queste, contemporaneamente capiremo quali siano giuridicamente autonome". ${ }^{52}$ Le Istituzioni procedono distinguendo i servi, i figli, naturali e adottivi, e nell'ambito dei liberi, definito in questo modo, separano ulteriormente le persone sottoposte a tutela e a curatela da quelle che non sono in nessuna di queste condizioni e che sono da ritenersi dunque, per negazione, le persone libere. ${ }^{53}$ Nello stesso modo procede anche Domingo de Soto, che si interroga nella sua Relectio sia sul dominio del padrone sui servi sia sulla relazione tra genitore e figli e tra coniugi, chiedendosi se esse possano essere formalizzate attraverso l'idea del dominio. ${ }^{54}$

In secondo luogo il dominio non può essere una disposizione su cose o persone del tutto indeterminata, ma è necessario stabilire quale sia la sua origine. Perché l'uomo può disporre delle cose e come può farsi loro signore? I bisogni della sussistenza, il fatto che l'essere umano necessiti di acqua e cibo e riparo, può spiegare il mero uso, ma non può chiarire le altre forme di dominio, dall'usufrutto fino alla piena proprietà, soprattutto non può spiegare la capacità dell'uomo di dominare su tutte le cose e su tutti gli animali della terra, appropriandosi di essi, come si dice in Genesi 1, 28.

Dall'essere umano stesso, dalla sua condizione, non può dunque derivare nessuna facoltà di disposizione sulle cose e l'unica possibilità è che l'uomo riceva tale capacità da qualcun altro. Ovviamente, solo Dio può essere la fonte di questa facoltà, ed effettivamente Dio è il signore dell'universo e, perciò, essendo il creatore di tutti gli enti, detiene pieno possesso su di essi. Come egli li ha creati, così può anche annichilirli, e come egli ha determinato la loro essenza attuale, così può sempre mutarla in tutto o in parte. ${ }^{55}$

Con la sua creazione, Dio introduce tuttavia un ordine nel mondo e fa sì che alcune cose siano in funzione di altre. Ogni ente è quindi da un lato

51 IUSTINIANUS IMPERATOR. Institutiones, lib. I, tit. 3 prooem., p. 11-2.

52 IUSTINIANUS IMPERATOR. Institutiones, lib. I, tit. 8, prooem., p. 17.

53 IUSTINIANUS IMPERATOR. Institutiones, lib. I, tit. 13, prooem., p. 25-26.

54 DOMINGO de Soto. Relección De dominio, An iuste homo hominis dominus esse possit, par. 24-6, p. 126-134; Nec patria potestas, nec manus mariti in vera dominii notione inveniri possunt, par. 37-9, p. 170-176

55 DOMINGO de Soto. Relección De dominio, Quibus convenit habere dominium, par. 16, p. 110 e par. 18, p. 112-113. 
sovraordinato e dall'altro subordinato a qualche altro, ma un solo ente dell'universo è superiore a tutti gli altri e non è sua volta subordinato a nessuno di essi, e tale è l'uomo, che gode di questa sua particolare posizione per la sua somiglianza con Dio. Per tale analogia egli partecipa di tutti gli attributi divini e deve godere di dominio sul mondo, nello stesso modo in cui Dio ha piena signoria sul suo creato.

Bisogna tuttavia considerare in che cosa consista quest'analogia che lega l'uomo a Dio. ${ }^{56}$ Qual è l'immagine di Dio che si conserva nell'uomo? Seguendo Tommaso d'Aquino, ${ }^{57}$ anche Soto riconosce che solo l'uomo può dirsi veramente padrone delle proprie azioni, perché agisce sulla base del libero arbitrio, il quale, a sua volta, è una facoltà della volontà e della ragione, una voluntas deliberata. $\mathrm{Ma}$, più che la volontà, il vero principio degli atti umani è la deliberatio rationis e perciò il riferimento al fine dell'uomo e alla sua conoscenza razionale è l'elemento fondamentale affinché si dia, in ultima istanza, dominio.

Sulla conoscenza, quale vero principio della relazione di signoria, aveva posto l'attenzione soprattutto Aristotele, ${ }^{58}$ le cui considerazioni ritornano quando Soto spiega in che modo il libero arbitrio sia essenziale per il dominio. Nessuno può infatti essere signore delle azioni altrui se prima non è signore delle proprie. Il fuoco non ha dominio sul calore perché non può scegliere se riscaldare o raffreddare, ma non può che seguire la propria natura. Ma per essere signore delle proprie azioni è necessario conoscere il fine e i mezzi dell'agire, come anche bisogna sapere di essere un agente libero. Essere signori di enti esterni implica una prestazione di conoscenza ancora maggiore perché l'agente che utilizza le cose per il proprio utile deve conoscere se stesso, il proprio utile, le cose esterne e ciò che esse possono apportare. ${ }^{59}$

L'uomo può perciò esercitare il suo dominio sulle altre creature perché è razionale, cioè intelligente, e l'immagine divina che egli conserva in sé, ovvero l'elemento di analogia tra umano e divino, è la sua anima razionale. Poiché egli comprende il mondo e vuole il bene del creato, può dominare su esso. Ovvero: la conoscenza (dell'ordine del mondo) è anche la fonte del dominio; l'uomo può asservire le altre creature a sé perché si conosce e le conosce. L'ultima giustificazione del dominio è dunque la consapevolezza, non tanto del mondo, ma dell'ordine che governa il mondo, che disegna tutti i possibili rapporti di dominio e che in ultima istanza risale a Dio, come al suo creatore.

56 Sulla fondazione analogica del dominio cfr. TELLKAMP. 2007, p. 158-162.

57 TOMÁS de Aquino. Summa theologiae, Ia IIae, quaest. 1, art. 1, resp., p. 556 $557^{\mathrm{b}}$.

58 ARISTOTELES. Politica, lib. I, cap. 1, 1252 ${ }^{\text {a }} 30-33$; cap. 2, 1253 ${ }^{\text {a }}$ 15-18; 31-32.

59 DOMINGO de Soto. Relección De dominio, Quibus convenit habere dominium, par. 10, p. 100. 
Il quadro nel quale si dispongono tutte le determinazioni dell'ordine, acquistando senso come parti di una stessa totalità, è la legge, che dunque è un riferimento necessario della dottrina del dominio. Effettivamente Soto avvia il suo percorso argomentativo presentando la legge ovvero le quattro diverse leggi, che descrivono quattro differenti livelli di ordine nel mondo; poi egli introduce la dottrina del dominio, con cui definisce negativamente la libertà e stabilisce quali siano le condizioni dell'azione giuridica e politica, e infine descrive nel dettaglio l'ordinamento giuridico. Lo schema fondamentale di Soto muove dalla constatazione che il diritto può essere inteso in due modi, o come regola della ragione, cioé come legge, o come oggetto del diritto, cioè come equità che la giustizia costituisce nei concreti rapporti umani. ${ }^{60} \mathrm{Il}$ passaggio tra le due parti è tuttavia dato da una dottrina della libertà umana definita negativamente attraverso la condizione delle persone non libere e in modo particolare attraverso la nozione del dominio, che vale anche come premessa generale per l'intera giustizia commutativa. Questo schema argomentativo, che collega legge e diritto attraverso la nozione del dominio e fa di quest'ultima l'origine della giustizia, risale a Soto ed è da considerare come un suo fondamentale contributo alla scuola di Salamanca e alla sistemazione della materia scolastica.

Il medesimo schema argomentativo si ritrova infatti anche nelle lezioni di Francisco de Vitoria, che riprendono gli stessi argomenti della Relectio de dominio discutendo la questione 62 della Secunda secundae, dedicata al tema della restituzione. Trattando il primo articolo, anche Vitoria concorda sul fatto che "aliqua sunt dicenda de dominio priusquam veniamus ad restitutionem" 61 e ricalca la stessa distribuzione degli argomenti proposta da Soto. Egli comincia trattando il nome del dominio, quindi affronta le tre diverse definizioni proposte dalla filosofia, dalla giurisprudenza e dalla teologia e ricava l'idea del dominio umano in una serie di dodici proposizioni che ritrovano il fondamento di questa nozione nella signoria di Dio sul suo creato e descrivono come da questa, attraverso il diritto umano, siano state divise le proprietà. Viene quindi discussa la facoltà di trasmettere la proprietà ad altri, che Soto, per mancanza di tempo, aveva tralasciato nella sua relectio. ${ }^{62}$ Vitoria conclude la sua trattazione con una dettagliata analisi dei casi singolari nei quali la trasmissione della proprietà può essere annullata dall'intervento del giudice. ${ }^{63}$

60 DOMINGO de Soto. De iustitia et iure libri decem, lib. I, Prooemium, p. 6a; lib. III, quaest. 1, art. 1, p. $191^{\text {a }}$.

61 FRANCISCO de Vitoria. De iustitia. Tomo primero, quaest. 62, art. 1, par. 4, p. 63.

62 DOMINGO de Soto. Relección De dominio, Conclusio, par. 42, p. 180.

63 FRANCISCO de Vitoria. De iustitia. Tomo primero, quaest. 62, art. 1, par. 4-54, p. 63-111. 
Il trattato De iustitia et iure di Soto ripropone infine in forma completa gli argomenti già toccati nella Relectio de dominio organizzandoli in uno schema ancora più lineare, rinunciando ai dettagli discussi da Vitoria e integrando le parti mancanti. La materia è qui strutturata in cinque questioni: il dominio in generale, gli oggetti del dominio, la divisione della proprietà, le forme particolari di dominio e la trasmissione della proprietà. Fondamentale è poi la circostanza che questo tema sia inserito come cerniera tra la discussione delle leggi e la dottrina della giustizia commutativa. In questa forma sistematica, datagli da Soto, e in questa posizione il trattato sul dominio fu conservato, tra gli altri, anche da Domingo Báñez, che diede risalto ancora maggiore all'autonomia di questa sezione argomentativa e la chiamò Preambolo sul dominio. ${ }^{64}$

\section{Riferimenti}

ALTHUSIUS, I. Politica methodice digesta atque exemplis sacris et profanis illustrata (1614). Aalen: Scientia, 1981.

ARISTOTELES. Ethica Nicomachea. Roma: Laterza, 2003.

Politik. Hamburg: Felix Meiner, 1990.

ARNISAEUS, H. De republica seu relectionis politicae libri duo. Francofurti [ad Viadrum]: Thymius, 1615. 1610.

. De iure maiestatis libri tres. Francofurti [ad Viadrum]: Sumtibus Thymius,

BALDINI, A. E. "Girolamo Frachetta informatore politico al servizio della Spagna". In: CONTINISIO, CH.; MOZZARELLI, C. Repubblica e virtù. Pensiero politico e Monarchia Cattolica fra XVI e XVII secolo. Roma: Bulzoni editore, 1995, p. 465-482.

BARRIENTOS GARCIA, J. Un siglo de moral economica en Salamanca (1526-1629): I: Francisco de Vitoria y Domingo de Soto. Salamanca: Ediciones Universidad de Salamanca, 1985, p. 139-278.

BELTRÁN DE HEREDIA, V. Domingo de Soto. Estudio biográfico documentado. Salamanca: Instituto de Cultura Hispánica, 1960, p. 117-73.

. "Introducción". In: FRANCISCO de Vitória. De iustitia. Tomo primero (2. 2. qq. 57-66). A cura di V. Beltrán de Heredia. Madrid: Publicaciones de la Asociación Francisco de Vitoria, 1934, p. IX-XII.

BERTELLONI, F. "Sobre la dispensabilidad de la ley antigua. La posición de Domingo de Soto a la luz de la tradición medieval". In: CRUZ CRUZ J. La ley natural come fundamento moral y jurídico en Domingo de Soto. Pamplona: EUNSA, 2007, p. 109-132.

BETTETINI, A. La restitutio in integrum processuale nel diritto canonico. Profili storicodogmatici. Padova, Cedam, 1994.

BINGHAM, C. James I of England. London: Weidenfeld and Nicolson, 1981.

64 DOMINGO Báñez. Decisiones de iure et iustitia, Praeambulum de dominio, p. 73$106[=108]$. 
BORNITZ, J. Discursus politicus de prudentia politica comparanda. Erphordiae: Birnstilius, 1602.

BOSE, J. A. De comparanda prudentia iuxta et eloquentia civili deque libris et scriptoribus ad eam rem maxime aptis dissertationes isagogicae (1677). Ienae: Bielkius, 1678, p. 38-39.

BRUFAU PRATS, J. "La noción analógica del dominium en Santo Tomás, Francisco de Vitoria y Domingo de Soto" (1957). In: ID. La Escuela de Salamanca ante el descubrimiento del Nuevo Mundo. Salamanca: San Esteban, 1989a, p. 11-47.

. "Domingo de Soto y su relección De dominio" (1966). In: ID. La Escuela de Salamanca ante el descubrimiento del Nuevo Mundo. Salamanca: San Esteban, 1989b, p. 79-101.

. "Preámbulo". In: DOMINGO de Soto. Relección De dominio. Granada: Universidad de Granada, 1964a, p. XXIII-XXIX.

. "Introducción". In: DOMINGO de Soto. Relección De dominio. Granada: Universidad de Granada, 1964b, p. 1-62;

CANO, M. De locis theologicis libri duodecim, Salmanticae, Gastius, 1563.

CAVARERO, A. "Giacomo I e il Parlamento". In: BIRAL, A. et al. Teorie politiche e Stato nell'epoca dell'assolutismo. Roma: Istituto Enciclopedia Italiana, 1980, p. 47-85.

COLER, CH. "De studio politico ordinando epistola". In: TACITUS, C.C. De situ, moribus et populis Germaniae libellus et in eum Christophori Coleri commentatio. Eiusdem De studio politico ordinando epistola. Hanoviae: Marnius et Aubrius, 1602, p. 88-117

COMPARATO, V. I. "Il pensiero politico della Controriforma e la ragion di Stato". In: ANDREATTA, A.; BALDINI A. E. Il pensiero politico dell'età moderna. Torino: Utet, 1999, p. 127-168.

CONTZEN, A. Politicorum libri decem. Moguntiae: Kinckius, 1621.

COVARRUBIAS Y LEYVA, D. DE. Variarum ex iure pontificio, regio et Caesareo resolutionum libri III (1552). Venetiis: Patessius, 1580.

. Regulae. Peccatum. De regulis iuris, lib. 6. Relectio (1554). Salmanticae: A Portonariis, 1558.

CRUZ CRUZ, J. "Introducción: la virtud de la justicia". In: DOMINGO Báñez. El derecho y la justicia. Decisiones de iure et iustitia. Pamplona: Ediciones Universidad de Navarra, [2008], p. 11-31.

DOMINGO Báñez. Decisiones de iure et iustitia (1594). Venetiis, Minima Societas, 1595.

DOMINGO de Soto. El abuso de los juramentos. La ocultación y revelación de secretos. Salamanca: San Esteban, 2000.

. Summulae (1554). Hildesheim, Georg Olms Verlag, 1980.

. De iustitia et iure libri decem (1553-1554). Madrid: Instituto de estudios políticos, 1967-8.

. Relección De dominio. Granada: Universidad de Granada, 1964.

. De cavendo iuramentorum abusu, ad laudem divini nominis institutio. Salmanticae: A Portonariis, 1551. 
FEENSTRA, R.; PERSENAIRE, C. E., "List of Sources". In: GROTIUS, H. De iure belli ac pacis libri tres (1625). Aalen: Scientia, 1993, p. 1027-1070.

FELLERMEIER, J. "Begriff und Verpflichtung des positivem Gesetzes bei Gabriel Vázquez". Scholastik. Vierteljahresschrift für Theologie und Philosophie, 15 (1940), p. 560-574.

FRANCISCO de Vitoria. De iustitia. Tomo primero (2. 2. qq. 57-66). A cura di V. Beltrán de Heredia. Madrid: Publicaciones de la Asociación Francisco de Vitoria, 1934.

FRANCISCO Suárez. De legibus (II 1-12). De lege naturali. Madrid: Consejo Superior de Investigaciones Científicas, 1974.

GIACON, C. La seconda scolastica 3. I problemi giuridico-politici. Suárez, Bellarmino, Mariana. Milano: Bocca, 1950.

GREENLEAF, W. H. "James I and the Divine Right of Kings". Political Studies, 5 (1957), p. 36-48.

GROTIUS, H. De iure praedae commentarius (1604-1605). Hagae Comitum: Nijhoff, 1868.

GUY, J. The Reign of Elizabeth I. Court and Culture in the Last Decade. Cambridge: CUP, 1995.

IUSTINIANUS IMPERATOR. Institutiones. In: NARDI, E. Istituzioni di diritto romano. Testi 2. Milano: Giuffrè, 1986.

LOVEJOY, A. La grande catena dell'essere (1936). Milano: Feltrinelli, 1966, p. 11-29.

. L'albero della conoscenza. Saggi di storia delle idee (1948). Bologna: Il Mulino, 1982, p. 29-41.

LUÍS de Molina. De iustitia et iure opera omnia (1602). Venetiis: Sessae, 1611.

KOSELLECK, R. "Einleitung". In: BRUNNER, O.; CONZE, W.; KOSELLECK, R. Geschichtliche Grundbegriffe. Historisches Lexikon zur politisch-sozialen Sprache in Deutschland. Stuttgart: Klett-Cotta, 1972, Bd. 1, p. XIII-XXVII.

MEARS, N. Queenship and Political Discourse in the Elizabethan Realms. Cambridge: CUP, 2005.

MEINECKE, F. L'idea della ragion di stato nella storia moderna (1924). Firenze: Sansoni, 1977, p. 1-22.

MEISNER, B. Dissertatio de legibus, in quatuor libellos distributa, quorum primus agit de lege in genere, secundus de lege aeterna, tertius de lege naturae, quartus de legibus humanis, tum politicis tum ecclesiasticis. Wittebergae: Raabe, 1616.

MUÑOZ DELGADO, V. Logica formal y filosofia en Domingo de Soto. Madrid: Publicaciones del Monasterio de Poyo, 1964, p. 27-59.

PEREÑA VICENTE, L. La Escuela de Salamanca. Proceso a la conquista de América. Salamanca: Caja de Ahorros y Monte de Piedad de Salamanca, 1986.

. "Estudio preliminar. La tesis de la paz dinámica". In: FRANCISCO de Vitoria. Relectio de iure belli o paz dinámica. Escuela Española de la Paz. Primera generación 1526-1560. Madrid: Consejo superior de investigaciones científicas, 1981, p. 27-94.

La Universidad de Salamanca, forja del pensamiento político español en el siglo XVI. Salamanca: Universidad de Salamanca, 1954. 
REIBSTEIN, E. Johannes Althusius als Fortsetzer der Schule von Salamanca. Untersuchungen zur Ideengeschichte des Rechtsstaates und zur altprotestantischen Naturrechtslehre. Karlsruhe: Müller, 1955, p. 17-53.

RISSE, W. "Einleitung”. In: DOMINGO de Soto. Summulae (1554). Hildesheim, Georg Olms Verlag, 1980, p. 3-8.

ROSSI, P. “Introduzione all'edizione italiana". In: LOVEJOY. L'albero della conoscenza. Saggi di storia delle idee (1948). Bologna: Il Mulino, 1982, p. 7-17.

SCATTOLA, M. “Sklaverei, Krieg und Recht. Die Vorlesung über die Regula 'Peccatum' von Diego de Covarrubias y Leyva". In: KAUFMANN, M.; SCHNEPF, R. Politische Metaphysik. Die Entstehung moderner Rechtskonzeptionen in der Spanischen Scholastik. Frankfurt am Main: Lang, 2007, p. 303-355.

. "Meinecke, Machiavelli e la ragion di stato". In: BASSANI, L. M.; VIVANTI C. Machiavelli nella storiografia e nel pensiero politico del XX secolo. Milano: Giuffrè, 2006a, p. 167-206.

. "Storia dei concetti e storia delle discipline politiche". Storia della storiografia, 49 (2006b), p. 95-124.

. Krieg des Wissens - Wissen des Krieges. Konflikt, Erfahrung und System der literarischen Gattungen am Beginn der Frühen Neuzeit. Padova: Unipress, 2006c, p. 22-55.

. “'Wie ein König im Krieg nach der wahrscheinlichen Meinung handeln soll'. Die Kriegslehre des Gabriel Vázquez im Horizont des Probabilismus". In: BRIESKORN, N.; RIEDENAUER, M. Suche nach Frieden: Politische Ethik in der Frühen Neuzeit III. Stuttgart: Kohlhammer, 2003, p. 119-153.

. L'ordine del sapere. La bibliografia politica tedesca del Seicento, numero monografico di Archivio della Ragion di Stato, 10-11 (2002-2003), p. 5-8.

. "Naturrecht als Rechtstheorie: Die Systematisierung der res scholastica in der Naturrechtslehre des Domingo de Soto". In: GRUNERT, F.; SEELMANN, K. Die Ordnung der Praxis. Neue Studien zur Spanischen Spätscholastik. Tübingen: Niemeyer, 2001, p. 21-47.

"Il concetto di tirannide nel pensiero politico tedesco della prima età moderna". Filosofia politica 10 (1996), p. 391-420.

SKINNER, Q. "Meaning and understanding in the history of ideas". In: ID. Visions of Politics. Volume 1: Regarding Method. Cambridge: CUP, 2002, p. 57-89.

. "Meaning and Understanding in the History of Ideas". History and Theory, 8 (1969), p. 3-53.

TELLKAMP, J. A. "Über den Zusammenhang von Freiheit und Sklaverei bei Vitoria und Soto". In: KAUFMANN, M.; SCHNEPF, R. Politische Metaphysik. Die Entstehung moderner Rechtskonzeptionen in der Spanischen Scholastik. Frankfurt am Main: Lang, 2007, p. 155-175.

THIEME, H. "Qu'est ce-que nous, les juristes, devons à la seconde scolastique espagnole?" (1973). In: THIEME, H. Ideengeschichte und Rechtsgeschichte. Gesammelte Schriften. Köln-Wien: Böhlau, 1986, Bd. 2, p. 908-922.

TODESCAN, F. Lex, natura, beatitudo. Il problema della legge nella scolastica spagnola del secolo XVI. Padova: Cedam, 1973, p. 83-157.

TOMÁS de Aquino. In primum[-quartum] Sententiarum. In: ID. Opera omnia. Stuttgart: Frommann-Holzboog, 1980. 
. Summa theologiae. Albae Pompeiae: Editiones Paulinae, 1988.

UNGEPAUR, E. Exercitia Iustinianea, sive disputationes ad IV. libros Institutionum imperatoris Iustiniani. Ienae: Lobenstein, 1650.

VÁZQUEZ MENCHACA, F. Controversiarum illustrium aliarumque usu frequentium libri tres (1564). Valladolid: Cuesta, 1931.

YATES, F. A. Astraea. The Imperial Theme in the Sixteenth Century. London: Routledge and Kegan Paul, 1975.

ZWIERLEIN, C. A., Discorso und Lex Dei. Die Entstehung neuer Denkrahmen im 16. Jahrhundert und die Wahrnehmung der französischen Religionskriege in Italien und Deutschland. Göttingen: Vandenhoeck und Ruprecht, 2006. 